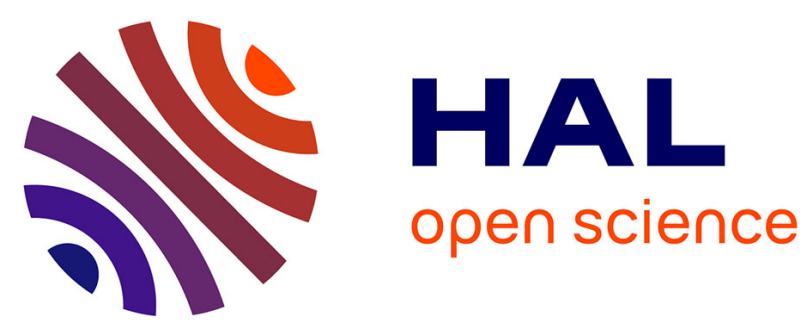

\title{
Implementing a flexible failure detector that expresses the confidence in the system
}

Anubis Graciela de Moraes Rossetto, Claudio R. Geyer, Luciana Arantes, Pierre Sens

\section{> To cite this version:}

Anubis Graciela de Moraes Rossetto, Claudio R. Geyer, Luciana Arantes, Pierre Sens. Implementing a flexible failure detector that expresses the confidence in the system. LADC 2016 - 7th Latin-American Symposium on Dependable Computing, Oct 2016, Cali, Colombia. hal-01352162

HAL Id: hal-01352162

https://hal.inria.fr/hal-01352162

Submitted on 27 Oct 2016

HAL is a multi-disciplinary open access archive for the deposit and dissemination of scientific research documents, whether they are published or not. The documents may come from teaching and research institutions in France or abroad, or from public or private research centers.
L'archive ouverte pluridisciplinaire HAL, est destinée au dépôt et à la diffusion de documents scientifiques de niveau recherche, publiés ou non, émanant des établissements d'enseignement et de recherche français ou étrangers, des laboratoires publics ou privés. 


\section{Implementing a flexible failure detector}

\author{
Anubis G. de M. Rossetto \\ Federal Inst. Sul-rio-grandense, Institute of Informatics Federal University \\ Passo Fundo, Brazil \\ Email: anubisrossetto@gmail.com
}

\author{
Luciana Arantes and Pierre Sens \\ Sorbonne Universités, \\ UPMC Univ. Paris 06, CNRS, \\ Inria, LIP6, Paris, France \\ Email: \{luciana.arantes, pierre.sens\}@lip6.fr
}

\begin{abstract}
Traditional unreliable failure detectors are per process oracles that provide a list of nodes suspected of having failed. In [1], we introduced the Impact failure detector that outputs a trust level value which is the degree of confidence in the system. An impact factor is assigned to each node and the trust level is equal to the sum of the impact factors of the nodes not suspected to have failed. An input threshold parameter defines an impact factor limit value, over which the confidence degree on the system is ensured. The impact factor indicates the relative importance of the process in the set $S$, while the threshold offers a degree of flexibility for failures and false suspicions. We propose in this article two different algorithms, based on query-response message rounds, that implement the Impact FD whose conceptions were tailored to satisfy the Impact FD's flexibility. The first one exploits the time-free message pattern approach while the second one considers a set of bounded timely responses. We also introduced the concept that a process can be $P S$-accessible (or $\diamond P S-$ accessible) which guarantees that the system $S$ will always (or eventually always) be trusted to this process as well as two properties, $P R(I T)$ and $P R(\diamond I T)$, that characterize the minimum necessary stability condition of $S$ that ensures confidence (or eventual confidence) on it. In both implementations, if the process that monitors $S$ is $P S-$ accessible or $\diamond P S-$ accessible, at every query round, it only waits (or eventually only waits) for a set of response that satisfy the threshold. A crucial facet of this set of processes is that it is not fixed, i.e., the set of processes can change at each round, which is in accordance with the flexibility capacity of the Impact FD.
\end{abstract}

\section{Introduction}

There are several important works in the literature concerning unreliable fault detectors (FDs). A FD can informally be seen as a per process oracle which, when invoked, provide information about processes liveness [2].

In [1], we introduced the Impact failure detector. In contrast with traditional unreliable failure detectors [3] [4] that output the set of nodes suspected of having failed, the Impact FD outputs a trust level concerning a given system $S$. The output can be considered as the degree of confidence in the system. To this end, an impact factor, that is defined by the user, is assigned to each node and the trust level is equal to the sum of the impact factors of the trusted nodes, i.e., those nodes not suspected of failure. Furthermore, an input threshold parameter defines an impact factor limit value, over which the confidence degree on $S$ is ensured. Hence, by comparing the trust level with the threshold, a process $p$ that monitors $S$ knows whether the system is trusted or not.

We consider "trusted" the expectation that a system will behave in a particular manner for a specific purpose even in the face of failures, i.e., the system is able to maintain the normal functionality. The impact factor indicates the relative importance of the process in the set $S$, while the threshold offers a degree of flexibility for failures and false suspicions, thus allowing a higher tolerance of instability in the system. For instance, in an unstable network, although there might be many false suspicions, depending on the value assigned to the threshold, the system can remain trustworthy. [5]

Having the above characteristics, the Impact FD can be applied for systems that have the following features: (1) applications that execute on them are interested on information about the reliability of the system as a whole and can tolerate a certain margin of failures. The latter may vary depending on the environment, situation, or context, such as systems that provide redundancy of software/hardware; (2) systems that organize nodes with some common characteristic into groups; (3) systems where the nodes can have different importance (relevance) or roles and, thus, their failures may have distinct impact on the system.

Among many, (1) wireless sensor networks (WSNs) that monitor environment conditions or (2) replicated servers that offer some quality of service (QoS) such as bandwidth or response time are two examples of systems where the Impact FD could be applied. In WSN used to collect environmental data, the monitored area can be divided into management zones in accordance with different characteristics. Each zone comprises sensors of different types (e.g., humidity control, temperature control, etc.) and the density of the sensors depends on the characteristics of each zone. That is, the number of sensors can be different for each type of sensor within a given zone. Furthermore, the redundancy of the sensors ensures both area coverage and connectivity in case of failure. Each management zone can thus be viewed as a single set which has sensors of the same type grouped into 
subsets. This grouping approach allows a threshold to be defined as being equal to the minimum number of sensors that each subset must have to keep the connectivity and application functioning all the time. In the second example, if the primary server that offers some quality of server fails, $n$ backup servers could replace it, provided that this set of servers, together, offer the same (or higher) quality of service than the primary one. In such a scenario, the impact factor value of the main server or the sum of the impact factors of the backup servers must be greater or equal than the threshold value. If this holds, the system will always be trusted, offering the expected QoS.

We denote flexibility property the capacity of the Impact FD to tolerate a certain margin of failures or false suspicions, i.e., its capacity of accepting different set of responses that lead to a trusted state of $S$. Thus, this article presents two different implementations for the Impact FD tailored to satisfy the flexibility property. Both of them use query-response message rounds. The first one is based on the time-free message pattern approach [6] which does not assume bounds on process and communication delays but the relative speed among messages. A process $p$ broadcasts a query message to the nodes of $S$ that it monitors and then waits for responses from $\alpha$ processes or from a set $Q$ of processes whose response satisfy the trust level. In the second approach, $p$ can receive responses to its broadcast query from a set of processes within a bounded delay (timely responses).

We also defined two properties, $P R(I T)$ and $P R(\diamond I T)$, that characterize the minimum necessary stability condition of $S$ that ensures confidence (or eventual confidence) on it. In other words, if $P R(I T)$ (resp., $P R(\diamond I T)$ ) holds, the system $S$ is always (resp., eventually always) trusted for the monitor process $p$.

Inspired in [7], we have introduced the concept that a process can be $P S-$ accessible or $\diamond P S-$ accessible: a correct process $p$ is $P S$-accessible (resp., $\diamond P S$-accessible) if every query broadcast by $p$ obtains from the beginning (resp., eventually) a set $Q$ of responses that satisfy the degree of confidence in $S$, i.e., the trust level of $S$ is greater or equal the threshold $d^{S}$ value. In the case of the message pattern approach, this property implies that a query broadcast by $p$ receives responses from a set of process $Q$ which satisfy threshold ${ }^{S}$ and these responses are always (resp., eventually always) winning responses, i.e., arrive before the other responses. In the case of the timer-based approach, there exists a set $Q$ of processes that satisfy the threshold $d^{S}$ and a query broadcast by $p$ always (or eventually always) receives timely (i.e., within a known bounded delay) responses from each process of $Q$. Interestingly that, in both implementations the set $Q$ of processes is not fixed, i.e., can be different at each query, which is in accordance with the flexibility property of the Impact FD.

This paper is structured as follows. Section 2 outlines some basic concepts of unreliable failure detectors while Section 3 describes the system model. Section 4 describes the Impact Failure Detector and its properties. Section 5 presents two algorithms for the implementation of the Im- pact FD as well as their proofs of correctness. In Section 6 we discuss some existing related work. Finally, Section 7 concludes the paper and outlines some of our planned future research work.

\section{Unreliable Failure Detectors}

An important abstraction for the development of fault tolerant distributed systems is the unreliable failure detector [2]. The aim of the latter is to encapsulate the uncertainty of the communication delay between two distributed entities.

An unreliable FD can be seen as an oracle that gives (not always correct) information about process failures (either trusted or suspected). It usually provides a list of processes suspected of having crashed. In addition, a failure detection system consists of local modules in which each machine may monitor a group or subgroup of system processes, or even be monitored by other detectors.

According to [8], unreliable FDs are so named because they can make mistakes (1) by erroneously suspecting a correct process (false suspicion), or (2) by not suspecting a process that has actually crashed. If the FD detects its mistake later, it corrects it. For instance, a FD can stop suspecting at time $t+1$, a process that it suspected at time $t$. Although the unreliable FDs can not accurately determine the real state of processes, using them increases knowledge about the processes of the system [2]. This means that the aim of an unreliable FD is to encapsulate the uncertainty of the communication delay between two distributed entities.

Unreliable failure detectors are characterized by two properties, completeness and accuracy, as defined in [2]. Completeness characterizes the failure detector's capability of suspecting faulty processes, while accuracy characterizes the failure detector's capability of not suspecting correct processes, i.e., restricts the mistakes that the failure detector can make. FDs are then classified according to two completeness properties and four accuracy properties [2].

Notice that the type of accuracy depends on the synchronism or stability of the network. For instance, a strong accuracy requires a synchronous system while an eventual strong one relies on a partially synchronous system which eventually ensures a bound for message transmission delays and processes' speed.

\subsection{Implementation of Failure Detectors}

The literature has several proposals for implementing unreliable failure detectors which usually exploit either a timer-based or a message-pattern approach.

In the timer-based strategy, FD implementations make use of timers to detect failures in processes. Every process $q$ periodically sends a control message (heartbeat) to process $p$ that is responsible for monitoring $q$. If $p$ does not receive such a message from $q$ after the expiration of a timer, it adds $q$ to its list of suspected processes.

The message-pattern strategy does not use any mechanism of timeout. In [6], the authors propose an implementation that uses a request-response mechanism. A process $p$ 
sends a $Q U E R Y$ message to $n$ nodes that it monitors and then waits for responses (RESPONSE message) from $\alpha$ processes ( $\alpha \leq n$, traditionally $\alpha=n-f$, where $f$ is the maximum number of failures). A query issued by $p$ ends when it has received $\alpha$ responses. The other responses, if any, are discarded and the respective processes are suspected of having failed. A process sends $Q U E R Y$ messages repeatedly if it has not failed. If, on the next request-response, $p$ receives a response from a suspected process $q$, then $p$ removes $q$ from its list of suspects. This approach considers the relative order for the receiving messages and that one (or a set of nodes) always (or after a time) answers faster to the $Q U E R Y$ than the other nodes.

\section{System Model}

In this work, we consider that there is one process by node (site) or sensor. Thus, the word process can mean either a node, a sensor, or a site. We consider a distributed system which consists of a finite set ${ }^{1}$ of processes $S=\left\{q_{1}, \ldots, q_{n}\right\}$ with $|S|=n$.

Processes communicate by sending and receiving messages over bi-directional reliable links, i.e., they do not lose, duplicate or corrupt messages and they never generate spurious messages. The network is assumed to be fully connected, i.e., $\Lambda=(p, q) \mid p, q \in S$.

The system model is asynchronous. It is important to point out that an asynchronous system is characterized by the absence of bounds on process speed and message delay. As result, it is impossible for a process to distinguish a crashed process from a process that is slow or a process which communication is slow. Thus, for the two proposed implementations, the asynchronous system is enriched with new assumptions about synchrony of the links or the relative speed among.

Processes fail by crashing and do not recover. We define a correct process the one which never fails during the whole execution; otherwise it is faulty. We assume the existence of some global time denoted $T$. A failure pattern is a function $F: T \rightarrow 2^{S}$, where $F(t)$ is the set of processes that have failed before or at time $t$. The function $\operatorname{correct}(F)$ denotes the set of correct processes, i.e., those that have never belonged to a failure pattern $(F)$, while faulty $(F)$ denotes the set of faulty processes, i.e., the complement of $\operatorname{correct}(F)$ with respect to $S$.

Besides $S$, we consider a correct process $p(p \in$ $\operatorname{correct}(F)$ ) that monitors $S$.

\section{Impact Failure Detector}

The Impact FD can be defined as an unreliable failure detector that provides an output related to the trust level with regard to a set of processes. If the trust level provided by the detector, is equal to, or greater than, a given threshold

\footnotetext{
${ }^{1}$ In this work, 'set' and 'multiset' are used interchangeably. Unlike a set, an element of a multiset can appear more than once. This allows different processes to have the same identity.
}

value, defined by the user, the confidence in the set of processes is ensured. We can thus say that the system is trusted. We denote FD $\left(I_{p}{ }^{S}\right)$ the Impact failure detector module of process $p$ that monitors system $S$. When invoked in $p$, the Impact FD $\left(I_{p}{ }^{S}\right)$ returns the trust_level $_{p}{ }^{S}$ value which expresses the confidence that $p$ has in the set $S$.

Each process $q \in S$ has an impact factor $\left(I_{q} \mid I_{q}>\right.$ $0: I_{q} \in \mathbb{R}$ ). Furthermore, set $S$ can be partitioned into $m$ disjoint subsets. Notice that the grouping feature of the Impact FD allows the processes of $S$ to be partitioned into disjoint subsets, in accordance with a particular criterion. For instance, in a scenario where there are different types of sensors, those of the same type can be gathered in the same subset. Let then $S^{*}=\left\{S_{1}^{*}, S_{2}^{*}, \ldots S_{m}^{*}\right\}$ be set $S$ partitioned into $m$ disjoint subsets where each $S_{i}^{*}$ is a set composed of the tuple $\langle i d, i m p a c t\rangle$, where $i d$ is a process identifier and impact is the value of the impact factor of the process in question.

$S^{*}=\left\{S_{1}^{*}, S_{2}^{*}, \ldots S_{m}^{*}\right\}$ is a set of processes of such that $\forall i, j, i \neq j, S_{i}^{*} \cap S_{j}^{*}=\emptyset$ and

$\bigcup\left\{q \mid\left\langle q, \_\right\rangle \in S_{i}^{*} ; 1 \leq i \leq m\right\}=S$

We denote trusted $_{p}{ }^{S}(t)=$ $\left\{\right.$ trusted $_{1}(t), \ldots$, trusted $\left._{m}(t)\right\}$, where each trusted $_{i}$ $(1 \leq i \leq m)$ contains the processes of $S_{i}^{*}$ that are not considered faulty by $p$ at $t \in T$. Similarly to $S_{i}^{*}$, each trusted $_{i}$ is composed of the tuple $\langle i d$, impact $\rangle$.

The trust level at $t \in T$ of processes $p \notin F(t)$ of $S$ is the denoted trust_level $_{p}{ }^{S}$ such that trust_level $_{p}{ }^{S}(t)=$ $\left\{\right.$ trust_level $_{i} \mid$ trust_level $_{i}=$ sum $_{\left(\text {trusted }_{i}(t)\right) ; 1 \leq i \leq}$ $m\}$ where the function sum(subset) returns the sum of the impact factor of all the elements of subset. In other words, the trust_level $_{p}{ }^{S}$ is a set that contains the trust level of each subset of $S^{*}$ expressing the confidence that $p$ has in $S$.

An acceptable margin of failures, denoted as the threshold ${ }^{S}$, characterizes the acceptable degree of failure flexibility in relation to set $S$. The threshold $S$ is adjusted to the minimum trust level required for each subset. i.e., it is defined as threshold ${ }^{S}=\left\{\right.$ threshold $_{1}, \ldots$, threshold $\left._{m}\right\}$

The threshold ${ }^{S}$ is used by the application to check the confidence on the processes of $S$. If, there is $s$ subsets of $S^{*}$ such that the trust_level $_{i}(t) \geq$ threshold $_{i}(1 \leq i \leq m), S$ is considered to be trusted at $t$ by $p$, i.e., the confidence of $p$ in $S$ has not been compromised; otherwise $S$ is considered not trusted by $p$ at $t$.

Two points should be highlighted: (1) both the impact factor and threshold ${ }^{S}$ render the estimation of the confidence in $S$ flexible. For instance, it is possible that some processes in $S$ might be faulty or suspected of being faulty but $S$ can still be trusted; (2) the Impact FD can be easily configured to adapt to the needs of the environment. The threshold $d^{S}$ can be tuned to provide a more restricted or softer monitoring.

In Table 1 , we consider a set $S$, where $S^{*}$ is composed by three subsets: $S_{1}, S_{2}$, and $S_{3}$ $\left(S^{*}=\left\{\left\{\left\langle q_{1}, 1\right\rangle,\left\langle q_{2}, 1\right\rangle,\left\langle q_{3}, 1\right\rangle\right\},\left\{\left\langle q_{4}, 2\right\rangle,\left\langle q_{5}, 2\right\rangle,\left\langle q_{6}, 2\right\rangle\right\}\right.\right.$, $\left.\left.\left\{\left\langle q_{7}, 3\right\rangle,\left\langle q_{8}, 3\right\rangle,\left\langle q_{9}, 3\right\rangle\right\}\right\}\right)$. The values of threshold ${ }^{S}$ define that the subsets $S_{1}, S_{2}$ and $S_{3}$ must have at least two 
correct processes. The table shows several possible outputs for FD $\left(I_{p}{ }^{S}\right)$ : the set $S$ is considered trusted whenever, for each subset $S_{i}^{*}$, trust_level $(t) \geq$ threshold $_{i}$.

TABLE 1. EXAMPLE OF FD $\left(I_{p}{ }^{S}\right)$ OUtPUt: $S^{*}$ HAS THREE SUBSETS

\begin{tabular}{|c|c|c|c|c|}
\hline $\mathbf{t}$ & $F(t)$ & trusted $_{p}{ }^{S}(t)$ & trust_level $_{p}{ }^{S}$ & Status \\
\hline 1 & $\left\{q_{2}\right\}$ & $\begin{array}{l}\left\{\left\{\left\langle q_{1}, 1\right\rangle,\left\langle q_{3}, 1\right\rangle\right\},\right. \\
\left\{\left\langle q_{4}, 2\right\rangle,\left\langle q_{5}, 2\right\rangle,\left\langle q_{6}, 2\right\rangle\right\}, \\
\left.\left\{\left\langle q_{7}, 3\right\rangle,\left\langle q_{8}, 3\right\rangle,\left\langle q_{9}, 3\right\rangle\right\}\right\}\end{array}$ & $\{2,6,9\}$ & Trusted \\
\hline 2 & $\left\{q_{2}, q_{5}\right\}$ & $\begin{array}{l}\left\{\left\{\left\langle q_{1}, 1\right\rangle,\left\langle q_{3}, 1\right\rangle\right\},\right. \\
\left\{\left\langle q_{4}, 2\right\rangle,\left\langle q_{6}, 2\right\rangle\right\}, \\
\left.\left\{\left\langle q_{7}, 3\right\rangle,\left\langle q_{8}, 3\right\rangle,\left\langle q_{9}, 3\right\rangle\right\}\right\}\end{array}$ & $\{2,4,9\}$ & Trusted \\
\hline 3 & $\left\{q_{2}, q_{5}, q_{6}\right\}$ & $\begin{array}{l}\left\{\left\{\left\langle q_{1}, 1\right\rangle,\left\langle q_{3}, 1\right\rangle\right\},\right. \\
\left\{\left\langle q_{4}, 2\right\rangle\right\}, \\
\left.\left\{\left\langle q_{7}, 3\right\rangle,\left\langle q_{8}, 3\right\rangle,\left\langle q_{9}, 3\right\rangle\right\}\right\} \\
\end{array}$ & $\{2,2,9\}$ & $\begin{array}{l}\text { Not } \\
\text { Trusted }\end{array}$ \\
\hline
\end{tabular}

\subsection{Flexibility of the Impact FD}

The flexibility of the Impact FD characterizes its capacity of accepting different set of responses that lead to a trusted state of $S$. Let $P S$ be the set that contains all possible subsets which satisfy a defined threshold:

$$
P S=
$$

TPowerSet $(S$, threshold $) \mid T$ PowerSet $(S$, threshold $)=$ $\times \operatorname{PowerSet}\left(S_{i}\right.$, threshold $\left._{i}\right)$

Initially, the TPowerSet function generates the power set ${ }^{2}$ for each subset $\left(S_{i}\right)$ of $S$. Then, only the subsets of $S_{i}$ whose sum of their parts is greater than, or equal to, threshold $i$ are selected. Following this, the Cartesian product is applied to generate all possible combinations ${ }^{3}$, i.e., all the subsets generated satisfy the threshold ${ }^{S}$.

Let's consider the following example:

$$
\begin{aligned}
& S^{*}=\left\{\left\{\left\langle q_{1}, 1\right\rangle,\left\langle q_{2}, 1\right\rangle\right\},\left\{\left\langle q_{3}, 1\right\rangle,\left\langle q_{4}, 1\right\rangle\right\},\left\{\left\langle q_{5}, 1\right\rangle,\left\langle q_{6}, 1\right\rangle\right\}\right\} \\
& \text { threshold }^{S}=\{1,1,1\} \\
& P S=\text { TPowerset }\left(S * \text {, threshold }{ }^{S}\right)
\end{aligned}
$$

PowerSet $\left(\mathrm{S}_{1}^{*}\right.$, threshold $\left._{1}\right)=\left\{\left\{\left\langle q_{1}, 1\right\rangle\right\},\left\{\left\langle q_{2}, 1\right\rangle\right\},\left\{\left\langle q_{1}, 1\right\rangle,\left\langle q_{2}, 1\right\rangle\right\}\right\}$ PowerSet $\left(\mathrm{S}_{2}^{*}\right.$, threshold $\left._{2}\right)=\left\{\left\{\left\langle q_{3}, 1\right\rangle\right\},\left\{\left\langle q_{4}, 1\right\rangle\right\},\left\{\left\langle q_{3}, 1\right\rangle,\left\langle q_{4}, 1\right\rangle\right\}\right\}$ PowerSet $\left(\mathrm{S}_{3}^{*}\right.$, threshold $\left._{3}\right)=\left\{\left\{\left\langle q_{5}, 1\right\rangle\right\},\left\{\left\langle q_{6}, 1\right\rangle\right\},\left\{\left\langle q_{5}, 1\right\rangle,\left\langle q_{6}, 1\right\rangle\right\}\right\}$ $\mathrm{PS}=\operatorname{PowerSet}\left(\mathrm{S}_{1}^{*}\right.$, threshold $\left._{1}\right) \times \operatorname{PowerSet}\left(\mathrm{S}_{2}^{*}\right.$, threshold $\left._{2}\right) \times$ PowerSet $\left(\mathrm{S}_{3}^{*}\right.$, threshold 3$)$

PS $=\operatorname{PowerSet}\left(S_{1}^{*}\right.$, threshold $\left._{1}\right) \times \operatorname{PowerSet}\left(S_{2}^{*}\right.$, threshold $\left._{2}\right) \times$ PowerSet $\left(\mathrm{S}_{3}^{*}\right.$, threshold $\left.{ }_{3}\right)$

$\mathrm{PS}=\left\{\left\{\left\{\left\langle q_{1}, 1\right\rangle\right\},\left\{\left\langle q_{3}, 1\right\rangle\right\},\left\{\left\langle q_{5}, 1\right\rangle\right\}\right\},\left\{\left\{\left\langle q_{1}, 1\right\rangle\right\},\left\{\left\langle q_{3}, 1\right\rangle\right\},\left\{\left\langle q_{6}, 1\right\rangle\right\}\right\}\right.$, $\left\{\left\{\left\langle q_{1}, 1\right\rangle\right\},\left\{\left\langle q_{3}, 1\right\rangle\right\},\left\{\left\langle q_{5}, 1\right\rangle,\left\langle q_{6}, 1\right\rangle\right\}\right\},\left\{\left\{\left\langle q_{1}, 1\right\rangle\right\},\left\{\left\langle q_{4}, 1\right\rangle\right\},\left\{\left\langle q_{5}, 1\right\rangle\right\}\right\}$, $\left\{\left\{\left\langle q_{1}, 1\right\rangle\right\},\left\{\left\langle q_{4}, 1\right\rangle\right\},\left\{\left\langle q_{6}, 1\right\rangle\right\}\right\},\left\{\left\{\left\langle q_{1}, 1\right\rangle\right\},\left\{\left\langle q_{4}, 1\right\rangle\right\},\left\{\left\langle q_{5}, 1\right\rangle,\left\langle q_{6}, 1\right\rangle\right\}\right\}$, $\left\{\left\{\left\langle q_{1}, 1\right\rangle\right\},\left\{\left\langle q_{3}, 1\right\rangle,\left\langle q_{4}, 1\right\rangle\right\},\left\{\left\langle q_{5}, 1\right\rangle\right\}\right\}$,

$\left\{\left\{\left\langle q_{1}, 1\right\rangle\right\},\left\{\left\langle q_{3}, 1\right\rangle,\left\langle q_{4}, 1\right\rangle\right\},\left\{\left\langle q_{6}, 1\right\rangle\right\}\right\}$,

$\left.\left\{\left\{\left\langle q_{1}, 1\right\rangle\right\},\left\{\left\langle q_{3}, 1\right\rangle,\left\langle q_{4}, 1\right\rangle\right\},\left\{\left\langle q_{5}, 1\right\rangle,\left\langle q_{6}, 1\right\rangle\right\}\right\}, \ldots\right\}$

\footnotetext{
${ }^{2}$ the power set of any set $S$ is the set of all subsets of $S$, including the empty set and $S$ itself

${ }^{3}$ The $\times S_{i}$ is a means of abbreviating the Cartesian Product when there are several sets, e.g. $\mathrm{S}_{1}^{*}, \mathrm{~S}_{2}^{*}, \mathrm{~S}_{3}^{*}\left(X=\left(S_{1}^{*}\right) \times\left(S_{2}^{*}\right) \times\left(S_{3}^{*}\right)\right)$
}

For instance, if trusted $_{p}{ }^{S}\left(t_{1}\right)$ = $\left\{\left\{\left\langle q_{1}, 1\right\rangle\right\},\left\{\left\langle q_{3}, 1\right\rangle\right\},\left\{\left\langle q_{5}, 1\right\rangle\right\}\right\}$ and trusted $_{p}{ }^{S}\left(t_{2}\right)=$ $\left\{\left\{\left\langle q_{1}, 1\right\rangle\right\},\left\{\left\langle q_{3}, 1\right\rangle,\left\langle q_{4}, 1\right\rangle\right\},\left\{\left\langle q_{6}, 1\right\rangle\right\}\right\}, \quad p$ considers that the system $S$ is trusted at both $t_{1}$ and $t_{2}$.

\subsection{Some properties}

Similarly to many existing works (see Section 6), we define some properties and process/link behaviors in order to introduce some synchrony on the asynchronous system.

Considering the set $P S$, which characterizes the flexibility of the Impact FD, we define the following properties:

Impact Threshold Property - PR(IT): For a failure detector of a correct process $p$, the set trusted $_{p}{ }^{S}$ is always a subset of $P S$.

$$
P R(I T) \equiv p \in \operatorname{correct}(F), \forall t \geq 0, \operatorname{trusted}_{p}{ }^{S}(t) \subset P S
$$

Eventual Impact Threshold Property - PR( $\diamond I T)$ : For a failure detector of a correct process $p$, there is a time after which the set trusted ${ }_{p}{ }^{S}$ is always a subset of $P S$.

$$
\begin{gathered}
P R(\diamond I T) \equiv \exists t \in T, p \in \operatorname{correct}(F), \forall t^{\prime} \geq \\
t, \text { trusted }_{p}{ }^{S}\left(t^{\prime}\right) \subset P S
\end{gathered}
$$

If $P R(I T)$ (resp., $P R(\diamond I T)$ ) holds, the system $S$ is always (resp., eventually always) trusted by $p$.

Inspired by the concept that a process is $\diamond f$-accessible proposed in [7] (see Section 6), we also define the concept of a $P S$-accessible and a $\diamond P S$-accessible process:

A process $p \in \operatorname{correct}(F)$ is $P S$-accessible (resp., $\checkmark P S-$ accessible) if every $Q U E R Y$ message broadcast by $p$ obtains from the beginning (resp., eventually) a set $Q$ of responses that satisfy the threshold ${ }^{S}$.

Thus, if $p$ is $P S$-accessible (resp., $\diamond P S-$ accessible), $P R(I T)$ (resp., $P R(\diamond I T)$ ) holds for $p$.

We have then the following definitions for the message pattern and timer-based approaches:

Message-pattern approach: Given a query issued by $p$, the set of first $R E S P$ messages received by $p$ to this query are denoted winning responses [6], [9].

A process $p$ is $P S$-accessible (resp., $\diamond P S-$ accessible) if for $\tau_{0} \geq 0$ (resp., $\exists \tau_{0}, \forall \tau \geq \tau_{0}$ ) there exists a set $Q$ of processes such that $Q \in P S$ and $p \notin Q(\tau)$ and a $Q U E R Y$ message broadcast by $p$ at $\tau$ receives $R E S P$ messages from processes of $Q(\tau)$ and these responses are always (resp., eventually always) winning responses.

Timer-based approach: A process $p \in \operatorname{correct}(F)$ is $P S$-accessible (resp., $\diamond P S-$ accessible) if for $\tau_{0} \geq 0$ (resp., $\exists \tau_{0}, \forall \tau \geq \tau_{0}$ ) there exists a set $Q$ of processes such that $Q \in P S$ and $p \notin Q(\tau)$ and a $Q U E R Y$ message broadcast by $p$ at $\tau$ receives a $R E S P$ message from each process of $Q(\tau)$ by time $\tau+\delta$. 
We should point out that in both definitions of $P S-$ accessible and $\diamond P S-$ accessible, the set $Q(\tau)$ is not fixed and can be different at distinct times which is in accordance with the flexibility property of the Impact FD.

\section{Implementations of Impact FD}

In this section we present two different implementations of the Impact FD: the first one is based on the messagepattern approach and the second one on the timer-based approach. Both of them use query-response message rounds and were conceived to exploit the flexibility capacity of the Impact FD.

We consider that the monitor process $p \in \operatorname{correct}(F)$ and $p \notin S$. It repeatedly issues queries by calling the primitive broadcast $(m)$ which sends a copy of the $Q U E R Y$ message over every link from $p$ to $q, \forall q \in S$. At each new round, $p$ broadcasts a $Q U E R Y$ message. The time interval between two consecutive rounds is finite and arbitrary (resp., bounded) for the message pattern (resp., timer-based) implementation. When process $q$ receives a $Q U E R Y$ message from $p, q$ confirms its reception with a RESP message.

The following local variables are used in both algorithms:

- $r_{p}$ : round counter of process $p$

- trusted: it is the set formed by $\left\{\right.$ trusted $_{1}, \ldots$, trusted $\left._{m}\right\}$ ( $m$ the number of subsets of $S)$, where each trusted $i(1 \leq i \leq m)$ contains the processes of $S_{i}$ that are not considered faulty by $p$. Each trusted $_{i}$ is a set composed of the tuple $\langle i d$, impact $\rangle$, where $i d$ is the process identifier and impact is the value of the impact factor of the process that belongs to the subset trusted . $_{\text {. }}$

- trust_level: the set that contains the trust level of each subset of processes;

- $P S$ : the set comprising all possible subsets formed by processes of $S$, where the sum of its elements is greater than, or equal to, the threshold ${ }^{S}$.

We also defined the function $A d d()$ which is used in both algorithms:

- $\operatorname{Add}($ set, subset, $\langle q$, impact $\rangle)$ : function that adds the process $q$, with impact factor impact to the subset subset of the set set provided that $q$ is not already in subset.

\subsection{Message-pattern Implementation}

Algorithm 1 presents the message pattern approach implementation of the Impact FD of process $p$ with respect to $S$.

Process $p$ receives as input the set of process of the system $S$, the number of subsets of $S^{*}(m)$, the threshold value of each subset of $S^{*}$ (the set threshold ${ }^{* *}$ ) and the maximum number of messages to wait $(\alpha)$. The latter is a set $\alpha=\left\{\alpha_{1}, \ldots, \alpha_{m}\right\}$, where each $\alpha_{i}$ corresponds to a threshold value for the number of messages to wait from the processes of subset $S_{i}^{*}$. For instance, if $f_{i}$ denotes the maximum number of failures of processes of subset $S_{i}^{*}$, $\alpha_{i} \leq\left|S_{i}^{*}\right|-f_{i}$ (for $i=1$ to $m$ ).

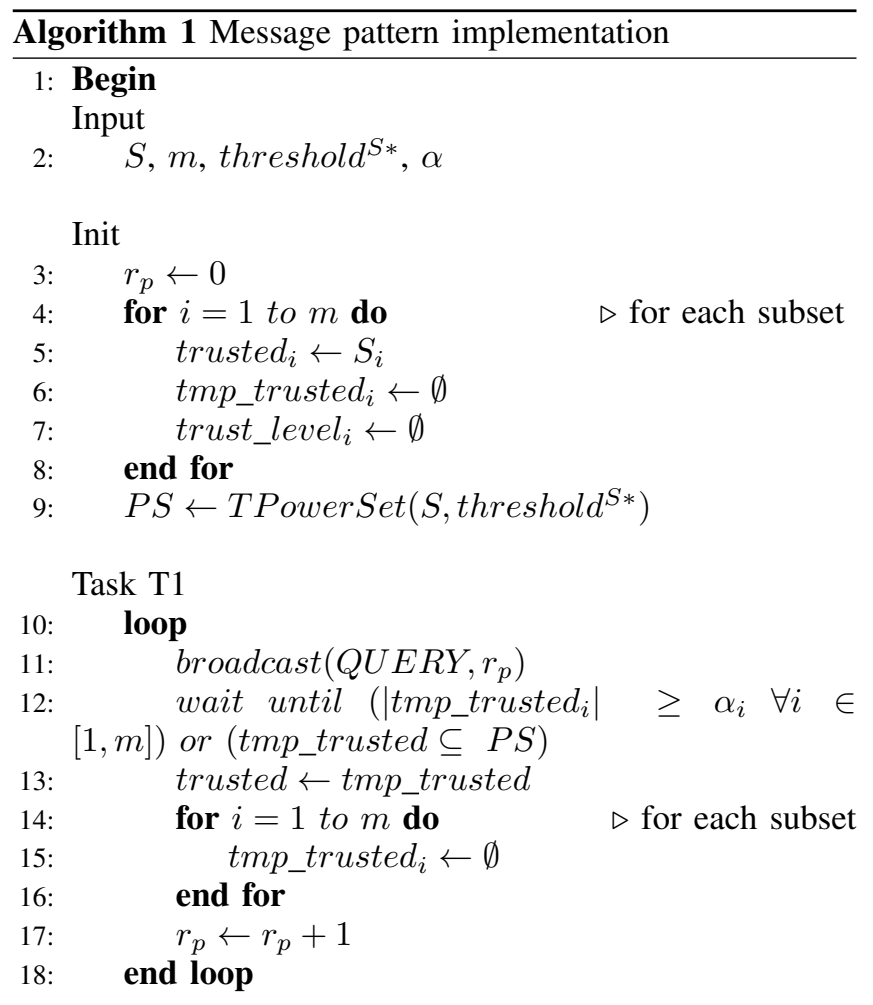

Task T2

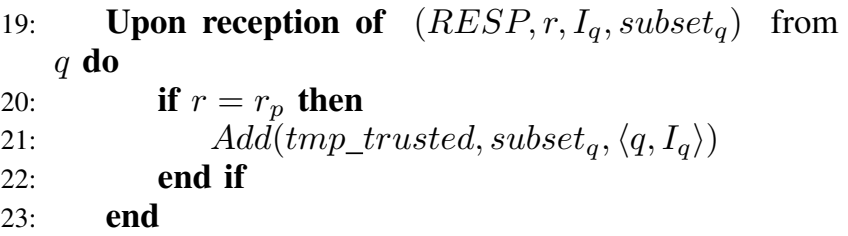

Task T3

24: Upon invocation of Impact() do

25: $\quad$ for $i=1$ to $m$ do $\quad \triangleright$ for each subset

26: $\quad$ trust_level $_{i} \leftarrow \operatorname{sum}_{\left(\text {trusted }_{i}\right)}$

27: $\quad$ end for

28: return trust_level

29: $\quad$ end

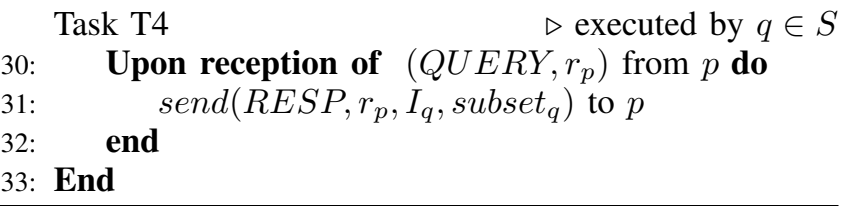

The algorithm has four tasks. Tasks T1, T2, and T3 are executed by $p$, while T4 is executes by $q \in S$.

At the initialization, trusted is initialized with the nodes of $S$. Then, the function TPowerSet is carried out to generate the set $P S$ which contains all possible subsets formed by processes of $S$ that satisfy the threshold ${ }^{S *}$. 
The variable tmp_trusted, at every query round, gathers the identification of processes that answered to the current query.

Task T1 of $p$ has an infinite loop. Firstly it sends the message $\left(Q U E R Y, r_{p}\right)$ to all processes of $S$ (line 11). Then, at each round $\left(\mathrm{r}_{p}\right), p$ waits for at least $\alpha_{i}$ responses $(1 \leq i \leq$ $m$ ) or until tmp_trusted is a subset of $P S$ (i.e., contains processes that satisfy the threshold ${ }^{*}$ ) (line 12). Finally, the round counter $\left(r_{p}\right)$ is incremented (line 17).

Task T2 handles the reception of messages (RESP, $r, I_{q}$ subset $_{q}$ ) sent by $q$. The message contains the round, the impact factor and the subset to which $q$ belongs. If round $r$ is equal to $r_{p}$, then $q$ is added to the trusted $_{q}$ set.

Task T3 handles the invocation of the Impact() function (line 24), which computes the trust_level of each subset and returns the trust_level of the trusted processes (line 28).

Task T4 is responsible for the reception of messages $Q U E R Y$ by process $q \in S$. When $q$ receives a (QUERY, $r$ ) message from the monitor process $p$ (line 30), $q$ must respond with a RESP message containing the round, its impact factor, and the number of its subset (line 31 ).

\section{Sketch of Proof}

Lemma 1. Process $p$ never blocks forever in a queryresponse round.

Proof. The only point that $p$ could block forever would be in the wait statement of Task T1 (line 12).

Let's consider round $r_{p}$ and that the system is blocked in the wait statement. Let's also suppose that the system is trusted in round $r$ and that the set of nodes included in tmp_trusted by Task T2 within round $r_{p}$ are also included in $P S$. In this case, the second condition of the wait becomes true and $T_{1}$ will not block. Let's now suppose that $p$ is blocked on thewait statement and that the second condition does not hold, i.e., $p$ will not be unblocked because of it. However, for every subset $S_{i}^{*}, p$ waits for $\alpha_{i}$ messages $\left(1 \leq i \leq m\right.$ ), where $f_{i}$ is the maximum number of processes of $S_{i}^{*}$ that can fail and $\alpha_{i} \leq\left|S_{i}^{*}\right|-f_{i}$. Therefore, as the channels are reliable and, even if $f_{i}$ nodes of each $S_{i}^{*}$ have failed, $p$ will receive $\alpha_{i}$ responses which will render the first condition true, and $p$ will be unblocked. In other words, since $\alpha_{i}$ is bounded by $n$ and $f_{i}$ and no query or response messages are lost, such a condition always ensures the progress of the failure detector.

Lemma 2. At every query $r_{p}$ issued by $p$, trusted tr $_{p}$ updated.

Proof. From Lemma 1, Task 1 never blocks and, thus, line 13 is always executed. This line is the only point where trusted is updated after initialization (line ??). Hence, at every query round, trusted $_{p}$ is updated.

Lemma 3. If $p$ is PS-accessible (resp., $\checkmark P S$-accessible), Algorithm 1 ensures that $P R(I T)$ (resp., $P R(\diamond I T)$ ) holds for $p$.

Proof. For $t=0$, trusted $p=S$. Let $\mathrm{Q}(\mathrm{r})$ be the set of winning responses and $r_{0}$ be the first round where
$Q\left(r_{0}\right) \in P S$. Thus, for $r_{0}$, trusted $=t m p \_t r u s t e d \subseteq P S$. Let then consider that $\forall r \geq r_{0}, Q(r) \in P S$ which characterize the $P S-$ accessibility of $p$. In this case, since from Lemma 2, trusted is updated at every query round, trusted $=$ tmp_trusted $\subseteq P S$. Let $t$ be the time when trusted is updated in round $r_{0}$ (line ?? or line 13). Therefore, $\forall t^{\prime} \geq t$, when the impact FD is invoked, $P R(I T)$ (resp., $P R(\diamond I T)$ ) holds.

Theorem 1. If $p$ is PS-accessible (resp., $\diamond P S-$ accessible), $\forall t \geq 0$ (resp. $\exists t^{\prime} \in T, \forall t \geq t$ ), trust_level $(t)_{p} \geq$ threshold.

Proof. The proof follows directly from Lemma 3.

\subsection{Timer-based Implementation}

Algorithm 2 shows a timer-based implementation of the Impact FD of process $p$ with respect to $S$.

For this implementation we added the assumption that there exists a known upper bound $\delta$ on the round-trip delay of messages, but it might not hold on all pairs of processes at all times. If $p \in \operatorname{correct}(F)$ is $P S$-accessible (resp., $\checkmark P S-$ accessible) it always (resp. eventually always) receives $R E S P$ messages from a set $Q$ of processes $Q \in P S$, in a delay of time smaller than $\delta$.

Process $p$ monitors the processes of $S$ and receives as input the set $S$, the number of subsets $m$, the threshold ${ }^{S *}$, the interval time $(\Delta>\delta)$ to send the broadcast message and the upper bound on the round-trip delay of messages $(\delta)$ for timely links.

The algorithm has five tasks. Tasks T1, T2, T3, and T5 are executed by $p$, while T4 is executes by $q \in S$.

At the initialization, each subset of trusted and trust_level is initialized as empty (lines 3 - 4). Then, the function TPower Set is carried out to generate the set $P S$ which contains all possible subsets formed by processes of $S$ that satisfy the threshold ${ }^{*}$ (line 7). The variables $r_{p}$, and timer timeout are also initialized.

At every round $r_{p}$, Task $\mathrm{T} 1$ of $p$ reset tmp_trusted and increments the round counter (lines 10-13). Periodically (interval of $\Delta$ time units), process $p$ sends to the processes in $S$ a $Q U E R Y$ message (line 14) and starts the timer timeout (line 15).

In Task $\mathrm{T} 2$, when process $p$ receives a $R E S P$ message sent by $q$, if round $r$ is equal to $r_{p}$ and $q$ is not in tmp_trusted, $q$ is added to tmp_trusted (lines 1718). At this point, if tmp_trusted is a subset of $P S$ (i.e., contains processes that satisfy the threshold ${ }^{S *}$ ) (line 20), then the variable tmp_trusted is assigned to trusted and the timeout is stopped.

Task T3 and T4 are the same of Algorithm 1 : task T3 handles the invocation of the Impact() function by $p$, which returns the sum of impact factor of the trusted processes (line 25); in Task T4, process $q \in S$ receives a (QUERY, $r)$ message from $p$ (line 31), $q$ answers with a RESP message containing the the round, its impact factor, and the number of its subset (line 32). 


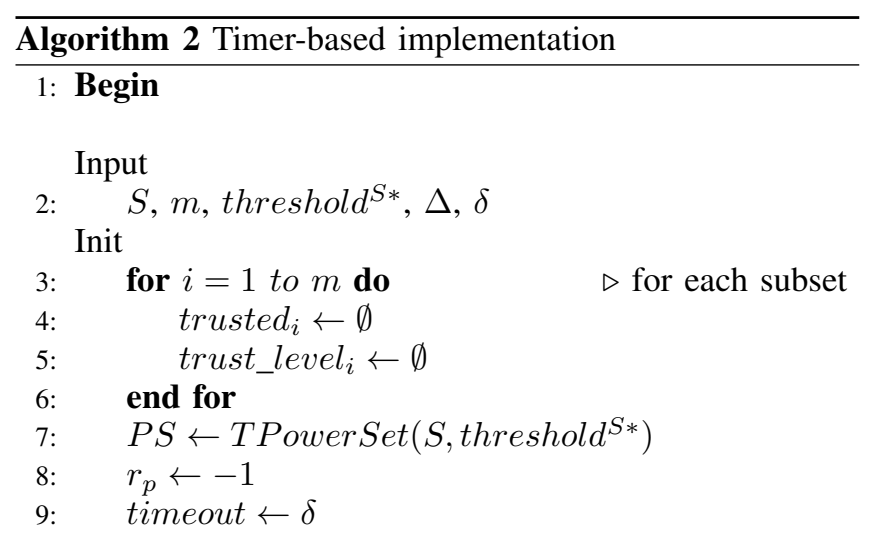

Task T1 - Repeat forever every $\Delta$ time unit

$$
\begin{aligned}
& \text { for } i=1 \text { to } m \text { do } \\
& \quad \text { tmp_trusted } \\
& \text { end for } \\
& r_{p} \leftarrow r_{p}+1 \\
& \text { broadcast }\left(Q U E R Y, r_{p}\right) \\
& \text { start timeout }
\end{aligned}
$$

Task T2

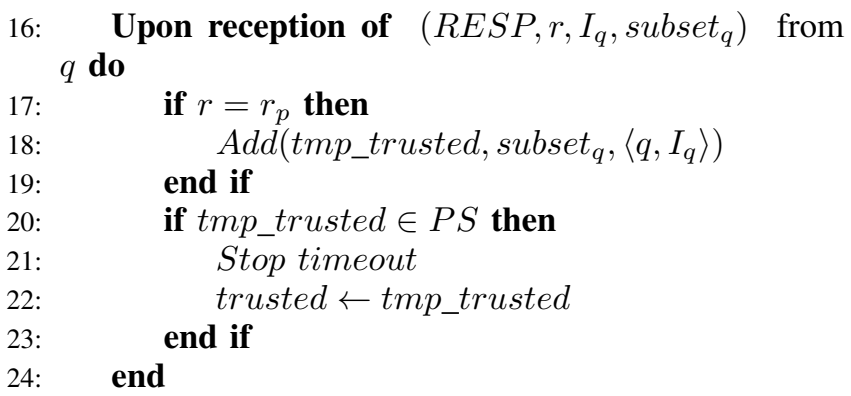

Task T3
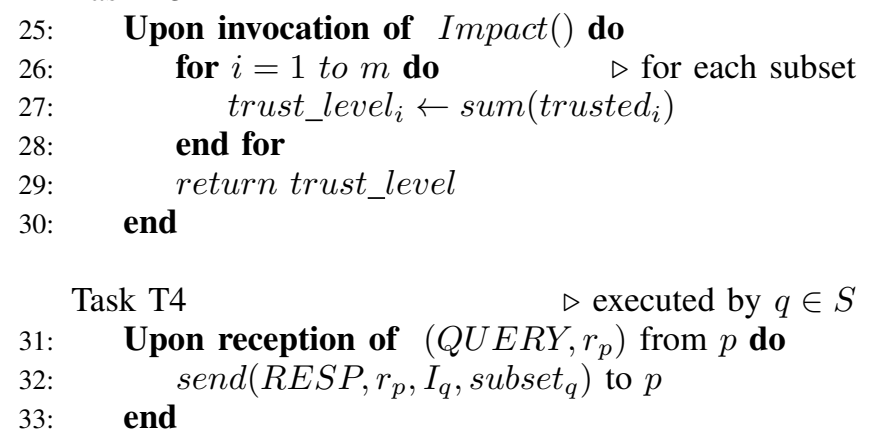

Task T5 - When timeout expires

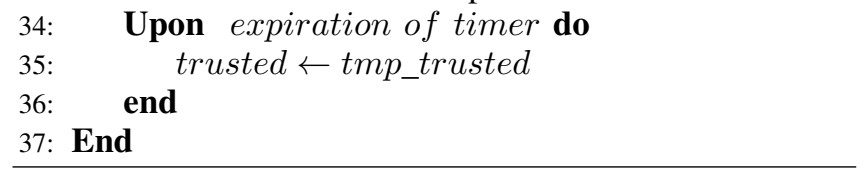

Upon expiration of the timer timeout (Task T4), $p$ assigns its current knowledge about trusted processes (in tmp_trusted) to trusted.

\section{Sketch of Proof}

Lemma 4. At every query $r_{p}$ issued by $p$, trusted ts $_{p}$ updated.

Proof. At every new query $r p$ in task T1, $p$ starts a timer (line 15). As $\Delta>\delta$, a new query will not be issued before the timer expires or is stopped. If the set of $Q$ messages received for this query are such that $Q \in P S$, the timer is stopped and trusted $_{p}=Q$ by Task T2 (lines 21 - 22). Otherwise, the timer will expires (Task T5) and trusted $_{p}$ will be updated with the set of processes that sent $R E S P$ messages to $p$ (line 35) within a delay of $\delta$.

Lemma 5. If $p$ is $P S$-accessible (resp., $\checkmark P S$-accessible), Algorithm 2 ensures that $P R(I T)$ (resp., $P R(\diamond I T)$ ) holds for $p$.

Proof. From Lemma 4, trusted $_{p}$ is updated at every query. Let $t_{0} \in T$ be the time where for every query issued by $p$ at $t \geq t_{0}$, a $Q U E R Y$ message broadcast by $p$ at $t$ receives a $R E S P$ message from processes of a $Q(t) \in P S$ within $t+\delta$. In this case, the timer started in every query at line 15 will never expire and, therefore, line 21 will be never executed $\forall t^{\prime} \geq t$. On the other hand, since $Q(t) \in P S$, the test of line 20 is always true. Hence $\forall t^{\prime} \geq t$, when the impact FD is invoked $P R(I T)$ (resp., $P R(\diamond I T)$ ) holds for $p$.

Theorem 2. If $p$ is PS-accessible (resp., $\diamond P S$-accessible), $\forall t \geq 0$ (resp. $\exists t^{\prime} \in T, \forall t \geq t$ ), trust_level $(t)_{p} \geq$ threshold.

Proof. The proof follows directly from Lemma 5.

\section{Related Work}

We can divide related studies of the literature into two groups: (1) unreliable failures detectors and (2) works which consider additional assumptions for asynchronous systems.

Unreliable failure detectors: Most of the unreliable fault detectors in the literature are based on a binary model and provide as output a set of process identifiers, which usually informs the set of processes currently suspected of having failed ( [2] [4]). However, in some detectors, such as class $\Sigma$ (resp., $\Omega$ ) [10], the output is the set of processes (resp., one process) which are (resp., is) not suspected of being faulty, i.e., trusted.

The $\phi$ Accrual failure detector [11] proposes an approach where the output is a suspicion level on a continuous scale, rather than providing information of a binary nature (trusted or suspected). It is based on an estimation of inter-arrival times which assuming that the latter follow a normal distribution. The suspicion level captures the degree of confidence with which a given process is believed to have crashed. If the process actually crashes, the value is 
guaranteed to accrue over time and tends toward infinity. In [12], the authors extended the Accrual FD by exploiting histogram density estimation. Taking into account a sampled inter-arrival time and the time of the last received heartbeat, the algorithm estimates the probability that no further heartbeat messages arrive from a given process, i.e., it has failed. The aim of Accrual failure detectors is to decouple monitoring from interpretation.

Starting from the premise that applications should have information about failures to take specific and suitable recovery actions, the work in [13] proposes a service to report faults to applications. The latter also encapsulates uncertainty which allows applications to proceed safely in the presence of doubt. The service provides status reports related to fault detection with an abstraction that describes the degree of uncertainty.

Considering that each node has a probability of being byzantine, a voting node redundancy approach is presented in [14] in order to improve reliability of distributed systems. Based on such probability values, the authors estimate the minimum number of machines that the system should have in order to provide a degree of reliability which is equal to or greater than a threshold value.

In [15], the authors propose the use of a reputation mechanism to implement failure detectors for large and dynamic networks. The reputation mechanism allows node cooperation through the sharing of views about other nodes. The proposed approach exploits information about the behavior of nodes to increase its quality in terms of detection. When classifying the behavior of the nodes, it includes a reputation service where the nodes periodically exchange heartbeat messages.

Additional assumptions for asynchronous systems: Several works have tried to circumvent the impossibility of the consensus in pure asynchronous systems in the presence of failures [16]. Therefore, the challenge is to identify properties that can be satisfied "nearly always" by the underlying asynchronous system enriched by some assumptions which allow an algorithm, for instance $\Omega$ (which can then be used to solve consensus in a system with a majority of correct processes) to be implemented during the "periods" in which the properties are satisfied [17].

The Message Pattern approach does not assume eventual bounds on process and communication delays. In [6], the authors consider that there is a correct process $p$ and a set $Q$ of $f$ processes (with $p \notin Q$, moreover, $Q$ can contain crashed processes) such that, each time a process $q \in Q$ broadcasts a query, it receives a response from $p$ among the first $(n-f)$ corresponding responses (such a response is called a winning response). Note that this assumption does not prevent message delays from always increasing without bound. This approach has been applied to the construction of a leader protocol.

Aguilera et al. introduced the $\diamond f-$ source assumption in [5] aiming at providing communication-efficient leader and consensus protocol implementations. In a system with $n$ nodes and up to $f$ process can crash, a $\diamond f-$ source node $p$ is a correct node with $f$ outgoing links that are eventually timely, i.e., there exist $t_{0}$ and a bound $\delta$, such that any message sent by $p$ after $t_{0}$ on one of these links is received at most $\delta$ units of time after it has been sent.

In [7], the authors introduce the notion of eventual $\diamond f$-accessible. A process $p$ is eventual $f$-accessible if there is a time $\tau_{0}$ such that, at any time $\tau \geq \tau_{0}$, there is a set $Q(\tau)$ of $f$ processes such that $p \notin Q(\tau)$ and a message broadcast by $p$ at $\tau$ receives a response from each process of $Q(\tau)$ by time $\tau+\delta$ (where $\delta$ is a bound known by the processes). This approach requires a majority of correct processes. Its interest lies in the fact that the set $Q$ of processes whose responses have to be received in a timely manner is not fixed and can be different at distinct times. The paper also presents a protocol building $\Omega$ when there is a process that is $\diamond f$-accessible forever, and all other links are fair-lossy.

In [18] and [19], the authors propose a model to implement unreliable FDs in dynamic networks with suitable assumptions for such a scenario. The message pattern model establishes conditions on the logical time the messages are delivered by processes. They present a stabilized responsiveness property $(S R P)$. The property states that there exists a time $t$ after which all nodes of $p_{i}$ 's neighborhood receive, to every of their queries, a response from $p_{i}$ which is always among the $\alpha_{j}$ responses to the query. That is, it denotes the ability of a node to reply to a query among the first nodes. Similarly to the winning channel approach, the response of $p_{i}$ is always a winning response.

\section{Conclusion and Future Work}

In this paper we have shown the flexibility capacity of our Impact FD provided by the impact factor and the threshold which enables the user to define the importance (e.g., degree of reliability) of each node and an acceptable margin of failures respectively.

We have defined two properties, $P R(I T)$ and $P R(\diamond I T)$, which denote the capacity of the Impact FD for accepting different set of responses that lead to a trusted state of the system $S$ as well as the concept of a $P S$-accessible (resp., $\diamond P S$-accessible) process such that every $Q U E R Y$ message sent by this process obtains from the beginning (resp., eventually) a set $Q$ of responses that satisfy the degree of confidence in $S$ (threshold th $_{S}$. Interestingly, that in both definitions of $P S$-accessible and $\checkmark P S$-accessible, the set $Q$ is not fixed and can be different at distinct times which is in accordance with the flexibility property of the Impact FD.

Then, we have presented two algorithms that implement the Impact FD tailored to exploit its flexibility feature. The first algorithm is based on a time-free message pattern approach which waits for responses from $\alpha$ processes or from a set $Q$ of processes whose responses satisfy the threshold ${ }^{S}$. The second algorithm assumes that for every query issued by $p$, the latter can receive timely responses. We have proved that, for both algorithms, if the monitoring 
process $p$ is $P S$-accessible (resp., $\diamond P S$-accessible) the system $S$ is always (eventually always) trusted by $p$.

As future work, we intend to extend the Impact FD so that it could be able to address dynamic impact factor values, i.e., they can vary during execution, which express the degree of reliability of the node (or reputation). We are also working on the reduction and equivalence of Impact FD in regard with other detectors (e.g., Sigma and Omega [13]), which will require some new assumptions and/or new definitions. In [1], we presented some performance evaluation results with a timer-based implementation of the Impact FD and traces collected from execution that we conducted on the PlanetLab platform [20] that include messages losses and failures of nodes. However, we still aim to conduct further performance experiments on different networks such as WiFi or LAN and, thus, comparing the Impact FD with other well-known failure detectors.

\section{References}

[1] A. G. Rossetto, C. F. Geyer, L. Arantes, and P. Sens, "A failure detector that gives information on the degree of confidence in the system," in 2015 IEEE Symposium on Computers and Communication, 2015, pp. 532-537.

[2] T. D. Chandra and S. Toueg, "Unreliable failure detectors for reliable distributed systems," Journal of the ACM (JACM), vol. 43, no. 2, pp. 225-267, 1996.

[3] W. Chen, S. Toueg, and M. K. Aguilera, "On the quality of service of failure detectors," Computers, IEEE Transactions on, vol. 51, no. 5, pp. 561-580, 2002.

[4] M. Bertier, O. Marin, P. Sens et al., "Performance analysis of a hierarchical failure detector." in DSN, vol. 3, 2003, pp. 635-644.

[5] M. K. Aguilera, C. Delporte-Gallet, H. Fauconnier, and S. Toueg, "Communication-efficient leader election and consensus with limited link synchrony," in Proceedings of the twenty-third annual ACM symposium on Principles of distributed computing. ACM, 2004, pp. 328-337.

[6] A. Mostefaoui, E. Mourgaya, and M. Raynal, "Asynchronous implementation of failure detectors," in 2013 43rd Annual IEEE/IFIP International Conference on Dependable Systems and Networks (DSN). IEEE Computer Society, 2003, pp. 351-351.

[7] D. Malkhi, F. Oprea, and L. Zhou, " meets paxos: Leader election and stability without eventual timely links," in Distributed Computing. Springer, 2005, pp. 199-213.

[8] N. Hayashibara, X. Défago, and T. Katayama, "Two-ways adaptive failure detection with the $\phi$-failure detector," in Workshop on Adaptive Distributed Systems (WADiS03). Citeseer, 2003, pp. 22-27.

[9] M. Raynal, "Eventual leader service in unreliable asynchronous systems: Why? how?" in Network Computing and Applications, 2007. NCA 2007. Sixth IEEE International Symposium on. IEEE, 2007, pp. 11-24.

[10] C. Delporte-Gallet, H. Fauconnier, R. Guerraoui, V. Hadzilacos, P. Kouznetsov, and S. Toueg, "The weakest failure detectors to solve certain fundamental problems in distributed computing," in Proceedings of the twenty-third annual ACM symposium on Principles of distributed computing. ACM, 2004, pp. 338-346.

[11] N. Hayashibara, X. Defago, R. Yared, and T. Katayama, "The $\varphi$ accrual failure detector," in Reliable Distributed Systems, 2004. Proceedings of the 23rd IEEE International Symposium on. IEEE, 2004, pp. 66-78.
[12] B. Satzger, A. Pietzowski, W. Trumler, and T. Ungerer, "A new adaptive accrual failure detector for dependable distributed systems," in Proceedings of the 2007 ACM symposium on Applied computing. ACM, 2007, pp. 551-555.

[13] J. B. Leners, T. Gupta, M. K. Aguilera, and M. Walfish, "Improving availability in distributed systems with failure informers," in Proc. of NSDI, 2013.

[14] Y. Brun, G. Edwards, J. Y. Bang, and N. Medvidovic, "Smart redundancy for distributed computation," in Distributed Computing Systems (ICDCS), 2011 31st International Conference on. IEEE, 2011, pp. $665-676$

[15] M. Véron, O. Marin, S. Monnet, and P. Sens, "Repfd-using reputation systems to detect failures in large dynamic networks," in 44th International Conference on Parallel Processing (ICPP-2015), 2015.

[16] M. J. Fischer, N. A. Lynch, and M. S. Paterson, "Impossibility of distributed consensus with one faulty process," Journal of the ACM (JACM), vol. 32, no. 2, pp. 374-382, 1985.

[17] A. F. Anta and M. Raynal, "From an asynchronous intermittent rotating star to an eventual leader," Parallel and Distributed Systems, IEEE Transactions on, vol. 21, no. 9, pp. 1290-1303, 2010.

[18] L. Arantes, F. Greve, P. Sens, and V. Simon, "Eventual leader election in evolving mobile networks," in Principles of Distributed Systems. Springer, 2013, pp. 23-37.

[19] F. Greve, P. Sens, L. Arantes, and V. Simon, "Eventually strong failure detector with unknown membership," The Computer Journal, vol. 55, no. 12, pp. 1507-1524, 2012.

[20] PlanetLab, "Planetlab," http://www.planet-lab.org, 2014, online. Acessado 16-setembro-2014. 